\title{
Development of carrier testing for common inborn errors of metabolism in the Wisconsin Plain population
}

\author{
Ashley Kuhl, CGC1,2, Sandra van Calcar, PhD, RD², Mei Baker, MD ${ }^{2,4}$, Christine M. Seroogy, MD2, \\ Gregory Rice, MD ${ }^{1,2}$ and Jessica Scott Schwoerer, MD ${ }^{1,2}$
}

\begin{abstract}
Purpose: This community project is an initiative through the University of Wisconsin Biochemical Genetics Clinic and the Wisconsin Newborn Screening Program to identify members of the Plain population who are at risk for having children with maple syrup urine disease (MSUD) or propionic acidemia (PA) or who have PA.

Methods: Because of the high prevalence of metabolic conditions in the Plain population and the importance of early intervention, a statewide outreach project was developed to provide targeted variant analysis of the common MSUD and PA pathogenic variants in this population through health-care provider distribution of blood spot testing kits. Awareness was achieved through outreach efforts with the state midwives guild and Plain population meetings.
\end{abstract}

Results: Eighty individuals were tested; diagnosis was confirmed for three adults with PA and one couple was identified as being at risk for having a child with PA. Genetic counseling was provided to those identified. Follow-up diagnostic testing was completed for the at-risk couple's children; none were found to be affected.

Conclusion: This initiative successfully provided accessible clinical testing for MSUD and PA for a high-risk population. Early identification of at-risk couples sets the foundation for early care of at-risk neonates, thereby improving future clinical outcomes.

Genet Med advance online publication 11 August 2016

Key Words: carrier testing; maple syrup urine disease; propionic acidemia; Plain population

\section{INTRODUCTION}

The Plain population represents "Christian groups that live simply, dress plainly, and live in the modern world but remain separate from it." ${ }^{\prime \prime}$ These communities originated from the Anabaptist movement in Europe in the 1500s. ${ }^{2}$ A small number of each group immigrated to the United States in the 1700s and 1800s and eventually settled in Wisconsin. In Wisconsin, the Plain population includes individuals from both the Old Order Amish and Mennonite communities. In 2014, the state's Plain population was approximately 20,000 , including approximately 17,025 $\mathrm{Amish}^{3}$ and 2,500 Mennonite members (D Kraybill, personal communication). Wisconsin's Amish population is the fourth largest in the United States ${ }^{3}$

As a result of religious separation and a restricted founder gene pool from subsequent consanguinity within these communities, there is a high prevalence of certain autosomalrecessive conditions, including several disorders currently detected by newborn screening programs (see Table 1).,1,4 Newborn screening is aimed at early identification of various genetic disorders, including several inborn errors of metabolism, and allows early treatment and thus improved long-term clinical outcomes. ${ }^{5}$ However, the Plain population's culture and way of life lead to several challenges in collecting newborn screens from neonates and treating those affected with identified disorders. Lack of medical follow-up due to financial (e.g., lack of medical insurance), logistical (e.g., the need to hire drivers to get to medical centers or home births that require a separate midwife visit for newborn collection), or cultural beliefs (e.g., the viewpoint that a physician need not be seen unless a child is seriously ill) is a common barrier. ${ }^{2}$

This project is an initiative through the University of Wisconsin Biochemical Genetics Clinic and the Wisconsin Newborn Screening Program to help families in the plain population identify family members who may be at risk for having a child with maple syrup urine disease (MSUD; OMIM 248600) or propionic acidemia (PA; OMIM 606054) or who may be at risk for having PA themselves. ${ }^{6}$ These conditions were chosen for this project because of their high prevalence in the Plain population as well as the importance of detection and the potential for early intervention for these life-threatening conditions. ${ }^{4}$

PA and MSUD are inborn errors of branched-chain amino acid metabolism caused by the deficiency of propionylCoA-carboxylase (PCC) and branched-chain dehydrogenase (BCKDHA), respectively, leading to the accumulation of amino acids and by-product organic acids. ${ }^{5}$ Each of these enzymes has multiple subunits, with variability in presentation associated with the subunit. ${ }^{5}$ In PA, reduced enzyme activity leads to an accumulation of propionic acid and its associated metabolites that are particularly toxic to the central nervous system and cardiac tissue. ${ }^{7}$ In MSUD, accumulation of all three

${ }^{1}$ Waisman Center, University of Wisconsin-Madison, Madison, Wisconsin, USA; ${ }^{2}$ Department of Pediatrics, University of Wisconsin-Madison, Madison, Wisconsin, USA;

${ }^{3}$ Department of Molecular and Medical Genetics, Oregon Health and Science University, Portland, Oregon, USA; ${ }^{4}$ Newborn Screen Lab at Wisconsin State Lab of Hygiene, Newborn Screen Lab Madison, Madison, Wisconsin, USA. Correspondence: Jessica Scott Schwoerer (jscottschwoerer@pediatrics.wisc.edu) 
Table 1 Metabolic newborn screening disorders more common in the Amish and Mennonite populations

\begin{tabular}{lll}
\hline Amish & \multicolumn{1}{c}{ Mennonite } & \multicolumn{1}{c}{ Both } \\
\hline Galactosemia & Tyrosinemia type 3 (2 pathogenic variants) & Phenylketonuria \\
Glutaric acidemia type 1 & Tyrosinemia type 1 & 3-Methyl crotonyl CoA carboxylase deficiency \\
Homocystinuria (due to & Phenylketonuria (2 pathogenic variants) & Propionic acidemia \\
methylenetetrahydrofolate reductase) & Medium-chain acyl CoA dehydrogenase deficiency & \\
& Maple syrup urine disease & \\
& Biotinidase deficiency & \\
\hline
\end{tabular}

Data from refs. 1,4.

branched-chain amino acids occurs, but elevations in leucine and 2-ketoisocaproic acid have particularly neurotoxic effects. ${ }^{5}$

In the Amish population, PA is caused by a common pathogenic variant, c. $1606 \mathrm{~A}>\mathrm{G}$, in the $\beta$-subunit of propionyl-CoA-carboxylase, $\mathrm{PCCB}$, and the clinical disease can present at any time during the life span. ${ }^{4}$ During the neonatal period, PA presents with poor feeding, lethargy, profound acidosis, and hyperammonemia. It can also present with metabolic decompensation in the setting of a stressor, often an intercurrent illness. During decompensation, all individuals with PA are prone to brain injury resulting in ataxia, lethargy, coma, and seizures. Long-term complications can include developmental delays, seizure disorder, and movement disorders due to basal ganglia injury. ${ }^{5}$ More recently, a cardiac phenotype with dilated cardiomyopathy, arrhythmia, and sudden death has been associated with PA..$^{8-14}$ The clinical phenotype most commonly seen in the Old Order Amish includes cardiac involvement, although metabolic decompensation and neurologic sequelae have also been described. In the Amish population, there are biochemically affected but seemingly clinically healthy individuals who have not had the symptoms associated with the neonatal presentations of PA. They are later recognized to have PA after the sudden cardiac death of a family member prompts testing (J.S.S, G.R., personal experience). Treatment includes diet; medications, including carnitine, biotin (PCC cofactor), and citrate; close cardiac monitoring; and standard care for cardiomyopathy and arrhythmia.

Unlike PA, MSUD in the Old Order Mennonite population presents most often during the neonatal period. With accumulation of leucine, the neonate can quickly become encephalopathic-initially presenting with poor feeding, lethargy, and eventually coma. Symptoms can develop at as early as $2-3$ days of life, often before routine newborn screening results are available. Immediate initiation of dietary therapy to reduce the leucine concentration is lifesaving; with long-term dietary and illness management, growth and developmental outcomes can be normal. ${ }^{5}$ Unlike the general population, in which the incidence of MSUD is $1 / 185,000$ (carrier frequency of $1 / 215$ ), approximately $1 / 10$ individuals in the Mennonite population carries a specific pathogenic variant $(\mathrm{c} .1312 \mathrm{~T}>\mathrm{A})$ in the BCKDHA that causes classic, severe disease. ${ }^{15}$
The carrier testing provided through this project enables identification of couples who are at risk for having an infant with one of these conditions as well as preparation for the birth of a potentially affected baby, including diagnosis by variant analysis before $12 \mathrm{~h}$ of life and immediate initiation of dietary treatment for an affected neonate. This identification of increased risk, through either carrier testing ${ }^{19}$ or a known affected family member, has been shown to preempt NICU stays (J.S.S, G.R., personal experience). Carrier testing also allows the identification of previously undiagnosed individuals with PA. This type of carrier testing has previously been performed for MSUD in the Old Order Mennonite population in Pennsylvania but has not previously been performed for PA.

\section{MATERIALS AND METHODS}

\section{Patients}

All Amish and Mennonite families residing in Wisconsin are eligible for MSUD carrier testing and PA carrier and diagnostic testing for the common pathogenic variants found in the Plain population. The general population and Plain families living outside Wisconsin are not eligible for this study. The testing is provided by the Wisconsin Newborn Screening Program and is offered free of charge as part of the services the program provides. This genetic testing is clinical testing and therefore did not require institutional review board approval for this study.

\section{Carrier testing kits}

The genetic testing was performed using kits that included specimen collection supplies as well as consumer-friendly, condition-specific information, provider collection and condition information, and patient consent forms (see Supplementary Information online).

For those who pursued testing, a blood sample obtained by finger prick was collected on a filter paper card, dried, and mailed to the Wisconsin State Laboratory of Hygiene for analysis. Targeted analysis for the common pathogenic variants found in the plain population for MSUD (BCKDHA c.1312T>A) and PA (PCCB c.1606A $>G$ ) was completed using a laboratorydeveloped test. ${ }^{16}$ Testing was ordered through a health-care provider but could be collected by a health-care provider or the family. Families were made aware that this testing did not 


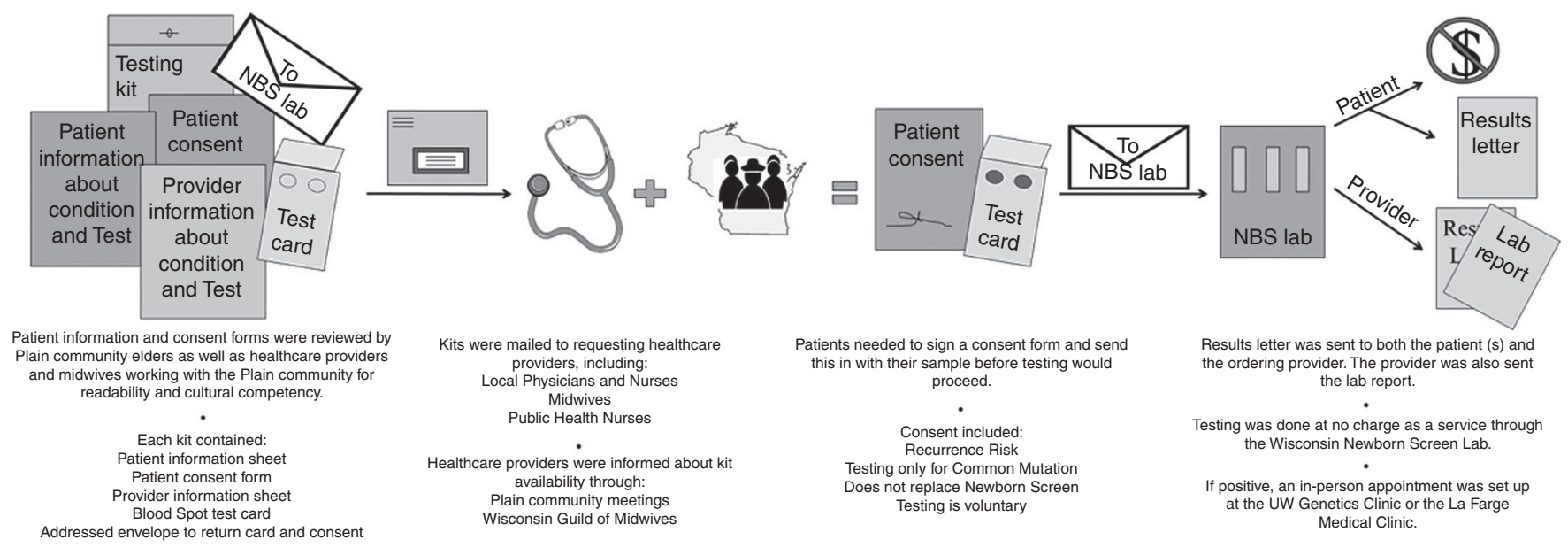

Figure 1 Flow diagram of the carrier testing process.

replace the standard newborn screen. A diagram of this process can be seen in Figure 1.

The Plain population does not provide formal education beyond the eighth grade and maintains different cultural norms than those outside of the Plain population regarding the discussion of certain topics, such as pregnancy. Thus, it was important that the material used to inform families about this testing and the testing results be clear, accurate, and culturally sensitive. All forms and information sheets were reviewed by metabolic geneticists, dietitians, genetic counselors, and a licensed midwife who follows patients in the Plain population.

\section{Outreach activities}

To increase accessibility, mail-order kits for both MSUD and PA testing were provided to midwives and other health-care providers following at-risk families. Kits were also distributed at Plain population meetings where clinical information about these conditions and the available testing was provided. Newborn screening education was also provided at the community meetings through physician-led discussions and midwife workshops to increase awareness of these disorders in the Plain population.

In a deliberate effort to build trust through community relationships, the family information for MSUD was also reviewed by a respected elder of a Wisconsin Plain community who has a family history of MSUD. The larger Wisconsin Partnership Program (WPP) project also includes community elders on its board, and their input was sought for the carrier testing project as well as the other projects associated with this grant (newborn screening awareness and provision of research-based testing for a wider range of clinical conditions in the Plain population).

To better understand and meet community needs, we utilized existing local relationships and the current infrastructure of the Wisconsin Newborn Screening Program and Wisconsin Midwife Guild. The majority of prenatal/perinatal care for the Plain population is provided by midwives (licensed and unlicensed birth attendants from the community referred to as lay midwives) and sometimes local physicians. Working closely with this group of care providers helped to establish trust with community members and facilitated effective and efficient access to and distribution of education materials, testing, and results.

\section{Patient communication}

Testing results were sent by mail to both the couple and the ordering provider. No letters were returned as undeliverable. Those who tested positive or were at increased risk for having a child with MSUD or PA were offered further counseling and medical care in an outreach clinic located in La Farge, Wisconsin, or at the University of Wisconsin Biochemical Genetics Clinic in Madison, Wisconsin. The results and kit distribution were tracked in an Excel spreadsheet.

\section{RESULTS}

\section{Distribution of kits}

From April 2014 to May 2015, 40 testing kits were distributed to four midwives (28 kits), one local physician (1 kit), and one public health nurse (11 kits). These Wisconsin health-care providers were from the cities of Withee, Blue River, Unity, Athens, and Eau Claire. Thirty-seven of these kits were utilized for testing. In addition, approximately 20 kits were used directly by University of Wisconsin Biochemical Genetics Clinic and La Farge Medical Clinic providers at outreach clinics and a community meeting. Nineteen of these kits were utilized for testing.

\section{Carrier testing findings}

Results were provided to 80 individuals as well as their midwives or local physicians (Table 2). Of these, three siblings in their early 20s were diagnosed with PA. For these individuals, the diagnosis of PA had been made with biochemical testing in infancy or early childhood but the family was lost to follow-up. They were returning for care and wanted to confirm the pathogenic variant for other family members. Similarly, an at-risk couple pursued testing because they had a son who died from suspected PA and wanted to know if their adult children were at risk. They were both determined to be carriers of PA. Testing 
Table 2 Results of testing over the course of 1 year

\begin{tabular}{lcc}
\hline & Propionic Acidemia & MSUD \\
\hline Molecular DNA testing completed & 32 & 48 \\
\hline Age in years (median age) & $5-70(26)$ & $16-49(29)$ \\
Known at-risk couple & $1^{\text {b }}$ & 0 \\
Type of testing & 17 & \\
$\quad$ Carrier & 15 & 48 \\
$\quad$ Diagnostic & 15 & 38 \\
Wild-type (no pathogenic variant) & 14 & 10 \\
Heterozygous (one pathogenic variant) & $3^{c}$ & 0 \\
\hline Homozygous (two pathogenic variants) & & \\
\hline
\end{tabular}

at-risk couple is a couple with both partners who are at least heterozygous for the common pathogenic variant; therefore, risk for an affected offspring is at least $25 \%$. ${ }^{b}$ Family identified as being at risk due to family history. ${ }^{\text {cPrior biochemical }}$ diagnosis as infant/young child and molecular DNA testing to confirm diagnosis. MSUD, maple syrup urine disease.

was subsequently provided to all nine of their adult children. None were found to have PA caused by the common pathogenic variant, but four were found to be carriers. Carrier testing was offered to their (future) spouses, and none of the tested spouses was found to be a carrier.

Because this testing looked only for these common pathogenic variants, negative results did not completely eliminate risk for the family or individual, but they did give a family and its health-care providers the ability to prepare for the possibility of an affected newborn. Given the possibility of adult-onset PA in the Amish population, variant analysis could also provide a diagnosis and allow initiation of treatment prior to the onset of symptoms.

\section{DISCUSSION}

Targeted carrier screening for genetic disorders in specific populations is not new and has been successfully completed in other populations. As an example, screening for Tay-Sachs disease in the Ashkenazi Jewish population has been shown to be successful. ${ }^{17}$ Using this example, various aspects need to be considered in developing a carrier screening project, including severity of the disease and availability of treatment, testing accuracy, costeffectiveness, funding for testing and genetic counseling, definition of the target population to be screened, development of a public/professional education program, informed consent for screening, and level of awareness in the community. ${ }^{18}$

The measure of success may vary depending on whether the disease is treatable. For the nontreatable Tay-Sachs disease, successful screening showed a dramatic decline in the incidence of the disease in the Ashkenazi Jewish population. ${ }^{19}$ For treatable metabolic diseases, however, preventing clinical presentation and need for hospitalization is a successful end point. This current approach of carrier screening and aggressive management of "at-risk" infants was initiated for infants at risk for MSUD at the Clinic for Special Children in Strasburg, Pennsylvania. ${ }^{19}$ Over 14 years, 21 at-risk neonates (identified by carrier testing or family history) were evaluated within $24 \mathrm{~h}$ and none required hospitalization. ${ }^{19}$ Without this identification, 23 other infants were identified; 20 had clinical presentations of critical illness requiring hospitalization. ${ }^{19}$

Because the common MSUD and PA pathogenic variants are site-specific in the Plain population, the testing and its results are straightforward. Molecular DNA testing for both MSUD and PA was already offered by the Wisconsin Newborn Screening Program, and utilizing this existing service enabled economical testing.

The needed outreach efforts in the Plains population indicate the importance of education and counseling for any populationbased testing program. ${ }^{20-22}$ Indeed, the educational component of this program was the key to its success. As part of a larger project funded by the Wisconsin Partnership Program, several community meetings hosted by the Plain population and attended by community elders, families, local physicians, and midwives were held and provided the opportunity to increase awareness of the disorders, the testing that was available, the benefits of newborn screening, and the treatability of many of these conditions. To encourage midwives to attend, training for the newborn screening process was provided. To establish trust and exemplify the benefit of this testing, panel discussions were held during the meeting and individuals with a family member who had either PA or MSUD spoke of their experience.

Communicating the testing information and results was a challenge because many Plain communities do not use or have limited access to certain forms of communication (e.g., phones, e-mail). Because of this limitation, information outside of meetings was communicated through health-care providers and word of mouth. It was unclear what background information the providers and community members had regarding genetic disorders. To overcome this challenge, we created provider and patient information sheets and sent the results letter to both the patients and the providers. We also provided a toll-free number for our clinic on the information sheets to help promote timely communication for those with phone access.

Although we did not diagnose any unsuspecting individuals or unsuspecting at-risk couples, plans were in place to follow up in person with any patients with abnormal results, at the outreach clinic in La Farge or the University of Wisconsin Clinics in Madison. The testing greatly lowered the estimation of occurrence risk for the couples who underwent carrier testing (from as high as $25 \%$ to less than $1 \%$ ) and promoted the importance of newborn screening to all who underwent this testing.

There are limited survey data about the view of genetic testing among the Amish and Mennonites, and much of it is based on clinicians' experience with the communities. A 1992 survey of Amish, Mennonites, and Hutterites about genetic testing for cystic fibrosis showed that it is important to understand cultural differences as well as variability in the attitudes for testing. ${ }^{23}$ This fits with our current experience with the Amish and Mennonites in that this population is heterogeneous in its view of Western medicine and the use of its services (unpublished data). For example, a survey performed through this Wisconsin 
Partnership Program found that more than $60 \%$ of the Plain families surveyed felt that newborn screening was encouraged or common within their community, whereas just under $25 \%$ were unsure about their community view about newborn screening or felt it was uncommon or (rarely) discouraged. ${ }^{24}$

This project illustrates the importance of developing relationships with health-care providers who work closely with Plain population members, community elders, and the community members themselves.

Future plans include improving the general communication of results and testing information through continued educational and training efforts with local health-care providers and midwives, continuing to work with the Plain population to improve our cultural competency in order to optimize the offerings of the current carrier and diagnostic testing, and expanding our test menu to include similar conditions that are common in this population, specifically, glutaric acidemia type 1 and galactosemia.

\section{SUPPLEMENTARY MATERIAL}

Supplementary material is linked to the online version of the paper at http://www.nature.com/gim

\section{ACKNOWLEDGMENTS}

We thank Catherine Reiser, Laura Birkeland, and Gretchen Spicer, for reviewing the manuscript. This study was funded, in part, by a Wisconsin Partnership Program Opportunity Grant.

\section{DISCLOSURE}

The authors declare no conflict of interest. The authors have full control of the primary data, which are available for review by the journal if requested.

\section{REFERENCES}

1. Strauss KA, Puffenberger EG. Genetics, medicine, and the Plain people. Annu Rev Genomics Hum Genet 2009; 10:513-536.

2. Hostetler, JA. The Amish, 3rd edn. Herald Press: Harrisburg, VA, 2013.

3. Young Center for Anabaptist and Pietist Studies, Elizabethtown College. Amish Population by State, 2014. http://www2.etown.edu/amishstudies/Population by state.asp. Accessed 5 September 2014.

4. Puffenberger EG. Genetic heritage of the Old Order Mennonites of southeastern Pennsylvania. Am J Med Genet C Semin Med Genet 2003;121C:18-31.
5. Saudubray J, van den Berghe G, Wlater JH (eds). Inborn Metabolic Diseases Diagnosis and Treatment, 5th edn. Springer: New York, 2012.

6. OMIM (Online Mendelian Inheritance in Man). Johns Hopkins University Center for Medical Genetics: Baltimore, MD, 1996. http://www3.ncbi.nlm.nih. gov/ omim/.

7. Pena L, Franks J, Chapman KA, et al. Natural history of propionic acidemia. Mol Genet Metab 2012;105:5-9.

8. Lee TM, Addonizio LJ, Barshop BA, Chung WK. Unusual presentation of propionic acidaemia as isolated cardiomyopathy. J Inherit Metab Dis 2009;32 Suppl 1:S97-101.

9. Laemmle A, Balmer C, Doell C, Sass JO, Häberle J, Baumgartner MR. Propionic acidemia in a previously healthy adolescent with acute onset of dilated cardiomyopathy. Eur J Pediatr 2014;173:971-974.

10. Jameson E, Walter J. Cardiac arrest secondary to long QT(C) in a child with propionic acidemia. Pediatr Cardiol 2008;29:969-970.

11. Kakavand B, Schroeder VA, Di Sessa TG. Coincidence of long QT syndrome and propionic acidemia. Pediatr Cardiol 2006;27:160-161.

12. Bhan AK, Brody C. Propionic acidemia: a rare cause of cardiomyopathy. Congest Heart Fail 2001;7:218-219.

13. Lücke T, Pérez-Cerdá C, Baumgartner M, et al. Propionic acidemia: unusual course with late onset and fatal outcome. Metabolism 2004;53:809-810.

14. Mardach R, Verity MA, Cederbaum SD. Clinical, pathological, and biochemical studies in a patient with propionic acidemia and fatal cardiomyopathy. Mol Genet Metab 2005:85:286-290.

15. Strauss KA, Puffenberger EG, Morton DH. Maple syrup urine disease. 30 January 2006 (updated 9 May 2013). In: Pagon RA, Adam MP, Ardinger HH, et al. (eds). GeneReviews. University of Washington: Seattle, WA.

16. Love-Gregory LD, Dyer JA, Grasela J, Hillman RE, Phillips CL. Carrier detection and rapid newborn diagnostic test for the common Y393N maple syrup urine disease allele by PCR-RFLP: culturally permissible testing in the Mennonite community. J Inherit Metab Dis 2001;24:393-403.

17. Vallance H, Ford J. Carrier testing for autosomal-recessive disorders. Crit Rev Clin Lab Sci 2003;40:473-497.

18. Kaplan F. Tay-Sachs disease carrier screening: a model for prevention of genetic disease. Genet Test 1998;2:271-292.

19. Morton DH, Morton CS, Strauss KA, et al. Pediatric medicine and the genetic disorders of the Amish and Mennonite people of Pennsylvania. Am J Med Genet C Semin Med Genet 2003;121C:5-17.

20. Falik-Zaccai TC, Kfir N, Frenkel P, et al. Population screening in a Druze community: the challenge and the reward. Genet Med 2008;10: 903-909.

21. Zlotogora J, Carmi R, Lev B, Shalev SA. A targeted population carrier screening program for severe and frequent genetic diseases in Israel. Eur I Hum Genet 2009;17:591-597.

22. Kaback M, Lopatequi J, Portuges AR, et al. Genetic screening in the Persian Jewish community: A pilot study. Genet Med 2010;12:628-633.

23. Miller SR, Schwartz RH. Attitudes toward genetic testing of Amish, Mennonite, and Hutterite families with cystic fibrosis. Am J Public Health 1992;82:236-242.

24. Sieren S, Grow M, GoodSmith M, et al. Cross-sectional survey on newborn screening in Wisconsin Amish and Mennonite communities. J Community Health 2016:41:282-288 